\title{
Robótica Educacional e Construcionismo como proposta metodológica para o desenvolvimento de ambientes de aprendizagem significativa
}

\author{
Jarles Tarsso Gomes Santos - CCEA/UEPB - jarlestarsso@ gmail.com \\ Jefferson Felipe Silva de Lima - CCEA/UEPB-CDSA/UFCG - \\ jfsilvadelima@gmail.com
}

Resumo. Este estudo tem como objetivo desenvolver atividades utilizando a Robótica Educacional, tendo como pressuposto metodológico as dimensões que formam a base da Teoria Construcionista proposta por Seymour Papert, de modo que todas as dimensões possam ser desempenhadas através de atividades para o desenvolvimento de protótipos, buscando entender as possibilidades da robótica no auxílio a problemas sociais e a sua capacidade para tornar a aprendizagem significativa. As atividades permitiram realizar práticas desafiadoras, em que o cognitivo do estudante foi requerido para a aquisição de habilidades importantes para a vida no século XXI. Foi identificado que as dimensões Construcionistas se caracterizam como um eficiente apoio metodológico capaz de auxiliar na aprendizagem do aluno.

Palavras-chave: Construcionismo. educação. robótica educacional. programação. protótipos.

\section{Educational Robotics and Construcionism as a methodological proposal for the meaningful learning environments}

\begin{abstract}
This study intents to develop activities using Educational Robotics having as methodological presupposition the dimensions of Construcionism proposed by Seymour Papert, so that its dimensions can be performed, through prototyping activities, looking for understand the possibilities that robotic can assist social problems and their possibilities to make the meaningful learning. The activities allowed doing challenging practices, that students' cognitive have been required for the development of important skills to life in the 21th century. It has been identified that dimensions of Construcionism are characterized as an efficient methodological support capable to assisting in student learning.
\end{abstract}

Keywords: Construcionism. education. educational robotics. programming. prototypes.

\section{INTRODUÇÃO}

A robótica se destaca como uma ciência e técnica da concepção, construção e utilização de robôs. Antes vista apenas como ficção, tornou-se uma das ferramentas mais importantes para a sociedade.

Com a popularização da Computação, a robótica passou a ser considerada uma ferramenta indispensável, aplicando-se a diversas áreas, contribuindo para a solução de problemas da sociedade, por meio de ações que são repetitivas e/ou cansativas ao ser humano (Santos, 2014). Após ser compreendida como uma ferramenta com potencial educativo, foi inserida em escolas de todo o mundo. Com isso, recebeu diversas denominações, sendo neste estudo tratada como Robótica Educacional (RE).

A RE configura-se como uma metodologia que pode ser usada em sala de aula, visando assegurar ao estudante uma forma de aprender aplicações que podem ser associadas ao conteúdo do currículo escolar (Santos, 2014). O processo de 
aprendizagem vinculado à $\mathrm{RE}$ acontece mediante uso de objetos mecânicos programáveis, adquiridos de empresas especializadas na confecção de kits robóticos ou por intermédio de materiais sucateados.

Apesar de um considerável número de escolas já possuírem recursos didáticos capazes de mudar a realidade do seu processo de ensino, é evidente a ausência de significado na aprendizagem do estudante e a grande dificuldade que ele encontra em assimilar os conteúdos. A aprendizagem significativa faz com que o aluno relacione conceitos já existentes em sua estrutura cognitiva, às novas ideias propostas pelo professor, desde que elas lhe façam sentido (Ausubel, 2000). O Construcionismo, por sua vez, permite o aprendizado por meio do fazer, induzindo o estudante a "pôr a mão na massa". Diante dessa construção de interesse, o estudante ganha motivação, que tem como consequência uma aprendizagem mais significativa (Valente, 1997).

Empenhado nessa perspectiva, o presente estudo propõe desenvolver atividades com auxílio da RE, tendo como pressuposto metodológico as cinco dimensões que formam a Teoria Construcionista proposta por Seymour Papert, buscando alcançar uma aprendizagem significativa, que resgate no estudante a motivação para a construção do seu conhecimento de forma autônoma, levando-o a pensar em problemas presentes no seu cotidiano e possibilidades para sua resolução. O público participante consiste em alunos matriculados no $1^{\circ}$ ano de um Curso Técnico em Informática, no ensino médio integrado à educação profissional, em uma escola no Sertão da Paraíba.

\section{ROBÓTICA EDUCACIONAL}

No final do século XX, a robótica já não era um objeto exclusivo da indústria. Nos anos 1980, a LEGO começou a produzir os primeiros kits para comercialização nas escolas, tornando a robótica acessível aos estudantes. Na mesma época, Seymour Papert (1928-2016) desenvolveu a linguagem LOGO, inicialmente proposta para ensino de programação para crianças e usada até hoje para ensinar àqueles com pouco conhecimento acerca da robótica e programação.

Hoje consolidada na educação, a RE passou a ser objeto de estudo em muitas escolas, tendo como principal foco montar, projetar e programar robôs vinculando a atividades educacionais (Almeida, Magalhães Netto e Custódio, 2017). As atividades podem ocorrer em ambientes de aprendizagem dentro ou fora da escola, demonstrando uma abordagem prática, que facilite a compreensão e motive o estudante a observar, abstrair e inventar (Zilli, 2004). Por meio da prática, é possível integrar outras áreas do conhecimento, desenvolvendo atividades interdisciplinares, nas quais os estudantes vivenciam conceitos anteriormente vistos em sala de aula (Bezerra et al., 2015).

O processo tendo como auxílio a robótica, implica no desenvolvimento de inúmeras habilidades comumente exigidas para um cidadão que vive no século XXI, como colaboração, resolução de problemas, exposição de pensamentos, autonomia e criatividade (Lessa et al., 2015).

\section{TEORIA CONSTRUCIONISTA}

A Teoria Construcionista foi desenvolvida por Seymour Papert, baseada na Teoria Construtivista de Jean Piaget (1896-1980). A teoria de Piaget aponta que o ser humano não nasce com um sistema cognitivo pronto, mas o constrói mediante interação com o meio físico e social, através das ações do próprio sujeito (Osti, 2009). 
O Construtivismo é uma proposta que permite a construção do conhecimento mediante a interação do sujeito com o meio em que ele está inserido (Becker, 1993). Diante disso, o professor propõe situações ao aprendiz, para que seu conhecimento seja elaborado, permitindo que ele encontre respostas para determinadas perguntas (Brousseau, 1996).

Papert e Piaget desenvolveram estudos juntos durante anos e a partir deles, Papert utilizou a ideia Construtivista para a idealização do Construcionismo, que possui como metodologia o aprendizado por intermédio do computador, onde o estudante constrói o seu próprio conhecimento auxiliado pela ferramenta digital (Papert, 1986).

Papert realizou diversos estudos até definir cinco dimensões que servem como base para a criação de ambientes de aprendizagem baseados no Construcionismo (Papert, 1986). Abaixo segue a descrição de cada uma das dimensões propostas:

- Dimensão pragmática: denota a ideia de que o conteúdo deve ser usado para fím prático, no qual o artefato desenvolvido seja utilizado em um curto período de tempo. Nela, o aprendiz tem a sensação de estar desenvolvendo algo que pode vir a ser útil.

- Dimensão sintônica: transmite ao aprendiz uma relação de sintonia com o conteúdo abordado. Para tal, é possível permitir ao aprendiz a escolha do tema proposto, fazendo com que o projeto se torne mais relevante, facilitando a relação aprendizprojeto e aumentando as chances de o conteúdo abordado ser melhor assimilado.

- Dimensão sintática: diz respeito à facilidade em que o aprendiz possui para acessar os elementos que formam o ambiente educativo, podendo avançar nos seus estudos usando o seu desenvolvimento cognitivo, sem a necessidade de pré-requisitos.

- Dimensão semântica: permite ao aprendiz interagir com elementos que tenham significado a ele, sem que a aprendizagem possua caráter formal.

- Dimensão social: traz a atividade ao cotidiano do aprendiz, de modo que o conteúdo interaja com a cultura do ambiente em que está sendo realizada a atividade.

Ao desenvolver atividades nas quais as dimensões propostas por Papert sejam estimuladas, são aumentadas consideravelmente as chances de que o conhecimento seja construído corretamente pelo aprendiz, desde que haja boa elaboração de atividades, para que possam abranger todas as dimensões propostas (Maltempi, 2000).

\section{MATERIAL E MÉTODOS}

Este trabalho apresenta uma metodologia de caráter empírico, havendo a conciliação do método qualitativo da pesquisa-ação e as dimensões que formam a Teoria Construcionista. Num primeiro momento, foi realizado um levantamento bibliográfico, buscando compreender conceitos e possibilidades das teorias utilizadas e como elas podem contribuir para o processo de aprendizagem do estudante.

Posteriormente, foi desenvolvida uma sequência didática para alcançar o objetivo central do estudo, observando as sugestões propostas pela pesquisa-ação. De acordo com Thiollent (2004) uma pesquisa pode ser definida como pesquisa-ação quando existir uma ação vinda das pessoas inseridas no contexto observado. Dentre algumas características dessa abordagem, está a interação entre pesquisador e as pessoas envolvidas no processo de investigação do objeto estudado, almejando a resolução do problema. Por meio da interação, há a seleção dos problemas que devem ser priorizados. 
Diante disso, atividades foram elaboradas, buscando corresponder as ideias propostas por cada dimensão Construcionista. As atividades constaram da identificação de problemas sociais observados pelos estudantes e num momento posterior, foi realizada a reflexão acerca das possibilidades que a robótica oferece para solucioná-los. Posteriormente, os estudantes desenvolveram alternativas para a solução dos problemas identificados, por meio da produção de protótipos robóticos. Os encontros para evidenciar os problemas, bem como as ações de intervenção ocorreram sempre na escola dos estudantes envolvidos, utilizando os laboratórios de robótica e informática.

Como método de coleta de dados para a análise dos resultados, foi utilizada a proposta da pesquisa-ação (Thiollent, 2004), na qual ocorre mediante questionamentos aos participantes envolvidos. Os questionários foram aplicados ao final de cada proposta de intervenção social dos estudantes, buscando compreender a consistência na aplicação das dimensões do Construcionismo.

\section{RESULTADOS E DISCUSSÃO}

As atividades iniciaram-se com a exploração do laboratório de robótica (Figura 1), que dispõe de material para o desenvolvimento de inúmeros modelos. A sequência didática desse momento aconteceu por meio da montagem de projetos para iniciantes, buscando alcançar níveis mais avançados progressivamente.

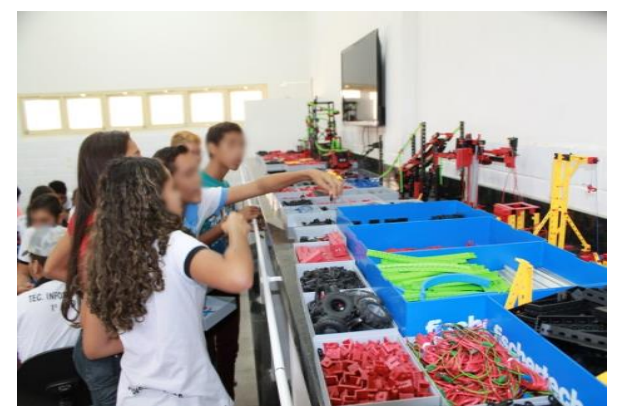

Figura 1 - Reconhecimento do laboratório de robótica.

A seguir, é possível observar o momento em que cada dimensão foi utilizada, avaliando suas contribuições pedagógicas e como auxiliaram para o desenvolvimento da sequência didática realizada neste estudo.

\subsection{DIMENSÃO SINTÁTICA}

As atividades envolvendo esta dimensão foram planejadas para despertar a curiosidade do estudante, mostrando a robótica como uma nova possibilidade de aprendizagem. A dimensão sintática facilita o acesso dos estudantes aos elementos básicos do contexto em que a proposta didática se aplica (Nunes e Santos, 2013). Por esse motivo, as primeiras atividades focaram na manipulação dos kits por intermédio dos manuais de orientação disponíveis em cada kit. Desse modo, cada estudante realizou a montagem de artefatos, mesmo sem conhecimento acerca da robótica.

\subsection{DIMENSÃO SINTÔNICA}

A dimensão sintônica estabelece que o aprendiz esteja em sintonia com a proposta apresentada, participando da escolha do elemento a ser desenvolvido (Nunes, 2016). A explanação dessa dimensão ocorreu com intuito de utilizá-la para definir quais projetos seriam desenvolvidos no decorrer das atividades, buscando instigar $o$ 
pensamento crítico do estudante para a investigação e resolução de problemas.

A sequência foi dividida em duas etapas, que ocorreram com (1) as sugestões de projetos e (2) o porquê ele deveria ser desenvolvido, observando sua relevância. Embasados em pesquisas e discussões, projetos foram selecionados, planejados e desenvolvidos e posteriormente nomeados e descritos, como é possível observar abaixo:

- Ventilador movido a energia solar: é um modelo robótico simples, que simula um ventilador alimentado por energia solar;

- Boné para deficientes visuais: consiste em um boné equipado com um sensor de distância e uma cigarra que aciona um sinal sonoro após a detecção de algum obstáculo pelo sensor de distância;

- RoboPharm: fornece a pessoas idosas o controle dos seus medicamentos diários, avisando o horário correto e qual medicamento deve ser tomado naquele instante, por meio da emissão de leds que apontam o reservatório correto.

A produção dos protótipos permitiu observar o processo motivacional gerado nos estudantes, que prontamente buscaram alternativas e soluções para eventuais problemas que surgiram durante o processo. É possível que o fato de permitir a livre escolha ocasione no discernimento acerca da importância do seu projeto, fazendo com que o estudante tenha mais entusiasmo para realizar a atividade.

\subsection{DIMENSÃO PRAGMÁTICA}

Nesta dimensão, o estudante observa a importância do seu projeto, arquitetando uma aplicabilidade para problemas atuais (Nunes e Santos, 2013). Diante dessa perspectiva, desafios surgem, necessitando que o estudante investigue novos conceitos que devem ser estimulados pelo seu cognitivo, buscando assimilar novos saberes (Nunes, 2016). Partindo dessa premissa, os estudantes elaboraram situações para uso dos seus projetos, identificando suas contribuições, por meio de testes e simulações.

\subsubsection{Ventilador movido a energia solar}

O ventilador movido a energia solar (Figura 2) propôs a ideia de economia de energia. Segundo a Empresa de Pesquisa Energética (EPE), no ano de 2017 o consumo de energia cresceu em todas as regiões do Brasil. O reflexo disso é o constante aumento nas contas de luz, que gera mais despesas aos consumidores. Os estudantes identificaram, por meio do estudo de alguns artigos, que utilizando energia solar é possível reduzir o consumo em aproximadamente $85 \%$ (Tsuruda, 2017).

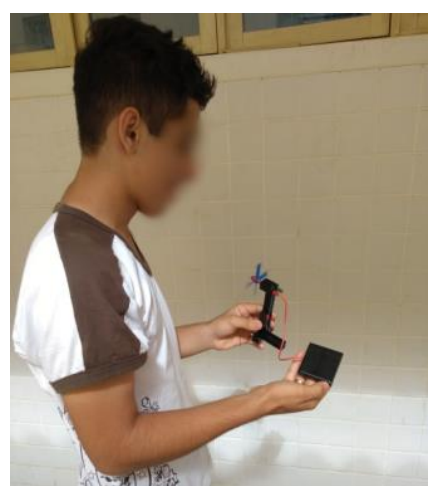

Figura 2 - Testes do ventilador movido a energia solar. 
O teste do dispositivo aconteceu em momento oportuno, para aproveitar a luz solar. Os responsáveis pelo teste foram questionados se o protótipo possui potencial para contribuir com a redução do gasto nas contas de luz em residências, caso torne-se um projeto real. O resultado foi unânime, em que todos concordaram que é uma proposta viável e que poderia ser implantada em todas as residências.

\subsubsection{Boné para deficientes visuais}

O boné para deficientes visuais (Figura 3) foi desenvolvido ao perceber a dificuldade que essa população possui ao caminhar entre determinados obstáculos que não estão ao alcance da bengala habitualmente usada por eles. $\mathrm{O}$ boné permitiu aos estudantes ter uma noção mínima da realidade de 3,6\% da população brasileira, segundo o Censo Demográfico do ano de 2010, realizado pelo IBGE. Com os olhos vendados, eles simularam o caminhar de um deficiente visual, operando primeiramente apenas a bengala e em seguida, com o protótipo desenvolvido por eles, observando se o boné pode facilitar os trajetos percorridos pelos deficientes.

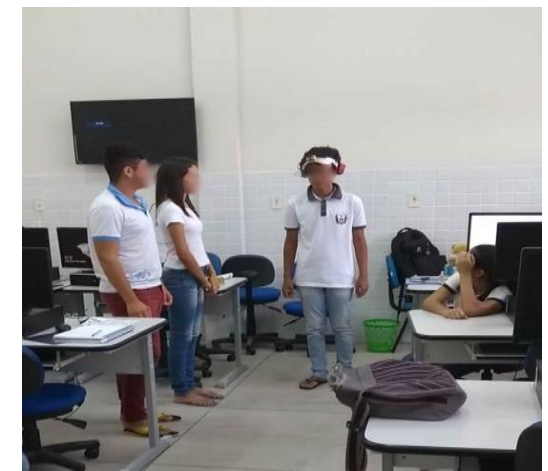

Figura 3 - Teste inicial, realizado para observar falhas no protótipo.

Para buscar melhorias, foi necessário realizar questionamentos aos estudantes que testaram o protótipo, almejando entender a sensação de utilizá-lo sem enxergar. O questionário foi aplicado a oito estudantes, sobre a possibilidade de o protótipo auxiliar um deficiente visual. $100 \%$ dos entrevistados afirmaram que sim, o protótipo pode auxiliar um deficiente. Entretanto, apenas 1 entrevistado (12,5\%) afirmou se sentir seguro em utilizar apenas o protótipo, sem auxílio de outro recurso, como uma bengala.

De acordo com Souza et al. (2016), um projeto dessa natureza pode beneficiar pessoas com deficiência visual, uma vez que permite a detecção de obstáculos com antecedência. Porém, de acordo com a opinião da maioria dos estudantes, o fato de não garantir com precisão o local onde se encontra o obstáculo, torna o boné inseguro.

\subsubsection{RoboPharm}

O RoboPharm tem por objetivo alertar pessoas idosas sobre o horário correto para tomar seus medicamentos e entregar-lhes o medicamento certo para o horário programado. O protótipo consiste em uma caixa equipada com um alarme sonoro e reservatórios para alocar os medicamentos. Cada reservatório possui leds instalados, que servem para indicar o reservatório que contém o medicamento correto. Por meio da programação do kit, é possível fazer com que o alarme soe em determinada hora do dia e o medicamento seja apontado por meio dos leds, indicando o reservatório correto. 
A ideia surgiu ao perceber a dificuldade dos idosos em controlar o horário dos seus remédios diários, havendo necessidade de constante supervisão. Dados colhidos do Sistema de Indicadores Sociais (SIS) mostram que os idosos representam cerca de $14,3 \%$ da população brasileira, com estimativa de um considerável crescimento para os próximos anos. Portanto, é importante atuar e discutir sobre esses problemas, propondo e pensando em possíveis soluções que sejam potencializadas por novas tecnologias.

Novamente o teste (Figura 4) foi acompanhado por oito estudantes, que observaram a efetividade do protótipo. Apenas um dos estudantes (12,5\%) não acreditou na possibilidade de um idoso operá-lo de forma autônoma. De acordo com Costa et al. (2016), a adaptação da população idosa é muito menor em relação ao público jovem quando o assunto é tecnologia. Mesmo com uma inovação capaz de melhorar a rotina da pessoa idosa, a dificuldade para manuseio de diversas tecnologias (e em muitas situações a ausência de orientação/acompanhamento) pode torná-la ineficiente.

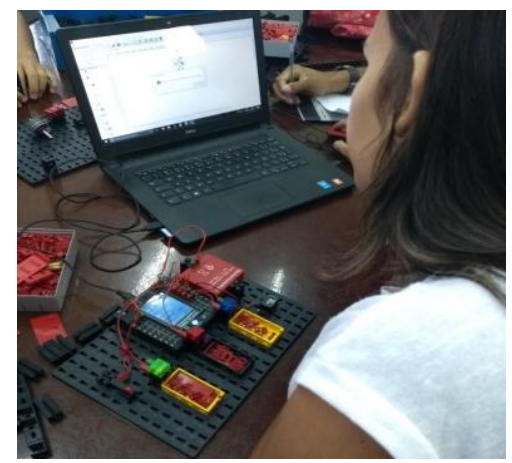

Figura 4 - Teste realizado para averiguar a efetividade do RoboPharm.

Quanto à crença em relação a possibilidade de auxílio a um idoso, todos acreditaram que o protótipo tem condições de facilitar a rotina desse público em particular. Todos os entrevistados também afirmaram confiar no uso do protótipo, possivelmente devido aos testes terem ocorrido com sucesso.

\subsection{DIMENSÃO SEMÂNTICA E SOCIAL}

A dimensão semântica busca levar ao estudante algo que tenha significado individual e coletivo, enquanto a dimensão social traz a ideia de que o conteúdo esteja em sincronia com suas relações interpessoais e seu cotidiano (Mota, 2014). Essas dimensões foram satisfeitas por meio dos projetos idealizados pelos estudantes, uma vez que o público ao qual foram oferecidos inclui pessoas do convívio deles.

Buscando entender o quão significativo sucedeu o desenvolvimento dos protótipos para os estudantes, foi necessário realizar um questionário fechado, composto por 5 perguntas, todas com opções de respostas SIM ou NÃO. O questionário foi respondido por um total de 18 estudantes, que participaram ativamente do processo de ideação, prototipação e testes dos protótipos.

A primeira pergunta questionou-os se a robótica pode oferecer auxílio a pessoas com alguma necessidade. Todos os entrevistados responderam que sim, sendo possível concluir que todos conseguiram de alguma forma entender o potencial oferecido pela robótica como instrumento capaz de solucionar problemas no mundo real.

A segunda pergunta questionou sobre a opinião dos estudantes acerca da eficiência dos projetos desenvolvidos. Novamente todos concordaram que os projetos 
podem cumprir o seu objetivo, com potencial para auxiliar o público ao qual se destina.

A terceira pergunta buscou entender quais estudantes se identificaram com os projetos desenvolvidos, ou seja, se conseguiram buscar significado no desenvolvimento. Apesar de todos afirmarem que os projetos são capazes de ajudar pessoas, parte deles $(27,8 \%)$ afirmou não ter encontrado tal significado. Durante o desenvolvimento de alguns projetos, foi possível ouvir comentários dos estudantes acerca de sujeitos que poderiam fazer uso dos protótipos, como parentes e amigos, podendo refletir a parcela que afirmou ter se identificado com algum dos projetos desenvolvidos $(72,2 \%)$.

A pergunta quatro questionou sobre a opinião dos estudantes acerca da possível melhoria dos protótipos. A grande maioria $(88,9 \%)$ afirmou que sim, todos necessitam de melhorias antes de ser considerado um produto final. Pelos testes realizados, ficou evidente essa necessidade, visto que falhas foram encontradas em algumas funções.

A última pergunta buscou entender a opinião dos estudantes quanto a sua vontade em continuar contribuindo para o desenvolvimento dos projetos. Uma pequena parte $(27,8 \%)$ afirmou não ter pretensão de continuar ajudando. Cabe destacar que esse número é idêntico ao de estudantes que afirmaram não ter se identificado com nenhum projeto, porém é incerto afirmar que os números tenham relação.

Cada dimensão que forma a base do Construcionismo foi estudada e aplicada nas atividades que deram embasamento a este estudo. É importante destacar a presença delas durante as atividades, observando quando cada uma foi satisfeita. Para melhor observar a relação dimensão $\mathrm{x}$ atividade, é descrito na Tabela 1 os protótipos desenvolvidos, junto às dimensões presentes durante o processo de cada um deles.

Tabela 1 - Presença das dimensões Construcionistas em cada etapa da pesquisa. (legenda: NC - não cumpriu, $\mathrm{C}$ - cumpriu e $\mathrm{CP}$ - cumpriu parcialmente)

\begin{tabular}{|l|c|c|c|c|c|}
\hline & & & & \\
Dimensão & Sintática & Sintônica & Pragmática & Semântica & Social \\
\hline Primeira manipulação dos kits & $\mathrm{C}$ & $\mathrm{NC}$ & $\mathrm{CP}$ & $\mathrm{CP}$ & $\mathrm{NC}$ \\
\hline Ventilador Solar & $\mathrm{CP}$ & $\mathrm{C}$ & $\mathrm{C}$ & $\mathrm{C}$ & $\mathrm{C}$ \\
\hline Boné para deficientes visuais & $\mathrm{NC}$ & $\mathrm{C}$ & $\mathrm{C}$ & $\mathrm{C}$ & $\mathrm{C}$ \\
\hline RoboPharm & $\mathrm{NC}$ & $\mathrm{C}$ & $\mathrm{C}$ & $\mathrm{C}$ & $\mathrm{C}$ \\
\hline
\end{tabular}

O início das atividades ocorreu com a primeira manipulação dos kits, em que os estudantes apenas seguiram instruções. Esse momento usou como método a dimensão sintática, sem necessidade de pré-requisitos. O ventilador solar também foi idealizado apenas seguindo instruções descritas em manuais. Entretanto, por exigir conceitos de programação, é possível afirmar que cumpriu apenas parcialmente a dimensão sintática.

O Boné para deficientes visuais e o RoboPharm, necessitaram de um aprofundamento de estudo por parte dos estudantes, pois envolveu bastante programação, contemplando assim as dimensões pragmática, devido seu potencial para aplicação; sintônica, pela sintonia observada entre estudante x projeto; semântica, quando o estudante entende a importância em auxiliar problemas reais e social, no momento em que o estudante auxilia sujeitos em seu entorno com suas ideias. 


\section{CONCLUSÕES}

O desenvolvimento dos protótipos permitiu entender o potencial oferecido pela robótica ante problemas reais, com materiais disponíveis no laboratório da escola. Foi possível aprender conceitos de linguagem de programação, mecânica, eletrônica, cuidados ao meio ambiente, bem como adquirir habilidades para o trabalho em equipe. Portanto, foi observada uma possível aplicação para a RE na educação básica.

As dimensões Construcionistas propostas por Papert tiveram grande relevância para o estudo, pois deram embasamento teórico para que fossem elaboradas as atividades. Elas mostraram-se suficientes para proporcionar uma aprendizagem significativa aos estudantes, norteando-os a adquirir o conhecimento de forma autônoma, por meio da interação com o ambiente da sala de aula. Diante das dimensões Construcionistas, foi possível integrar o conteúdo à realidade dos envolvidos, proporcionando uma aprendizagem motivadora, onde os estudantes identificaram as aplicações para o conhecimento adquirido.

Todos os projetos foram idealizados e montados em forma de protótipo, podendo estar longe de um produto final. Os testes realizados não levaram em consideração a opinião do público para o qual ele foi desenvolvido, de modo que a eficiência de cada projeto é de caráter duvidoso. Pretende-se desenvolver no futuro, estudos para o aprimoramento dos protótipos, para que sejam projetados levando em consideração os requisitos e embasamentos teóricos adequados, bem como a realização de testes pelos sujeitos que serão diretamente beneficiados por cada protótipo desenvolvido.

\section{REFERÊNCIAS BIBLIOGRÁFICAS}

ALMEIDA, T. O.; MAGALHÃES NETTO, J. F.; CUSTÓDIO, T. P. Desenvolvimento e Configuração de Cenários de Robótica para fomentar a Aprendizagem de Programação aos Alunos do Ensino Fundamental. In: WORKSHOP DE INFORMÁTICA NA ESCOLA, 23., 2017, Recife. Anais. Recife: Sociedade Brasileira de Computação, 2017, p. 205-214.

AUSUBEL, D.P. The acquisition and retention of knowledge: a cognitive view. Berlim: Springer Science, 2000.

BECKER, F. Modelos pedagógicos e modelos epistemológicos. Educação e Realidade, Porto Alegre. v. 19, n.1, p. 43-52, jan./jun., 1993.

BEZERRA NETO, R.P.; ROCHA, D. P.; SANTANA, A. M.; SOUZA, A. A. S. Robótica na Educação: Uma Revisão Sistemática dos Últimos 10 Anos. In: SIMPÓSIO BRASILEIRO DE INFORMÁTICA NA EDUCAÇÃO, 26., 2015, Maceió. Anais. Maceió: Sociedade Brasileira de Computação, 2015, p. 386-393.

BROUSSEAU, G. Os diferentes papéis do professor. In: PARRA C.; SAIZ, I. (Orgs.). Didática da matemática: reflexões psicopedagógicas. Tradução: J. A. Llorens. Porto Alegre: Artes Médicas, 1996. p. 48-72.

COSTA, N. P.; POLARO, S. H. I.; VAHL, E. A. C.; GONÇALVES, L. H. T. Storytelling: a care technology in continuing education for active ageing. Rev. Bras. Enferm, Brasília. v. 69, n. 6, nov./dec., 2016. 
LESSA, V. E.; FORIGO, F. M.; TEIXEIRA, A. C.; LICKS, G. P. Programação de Computadores e Robótica Educativa na Escola: tendências evidenciadas nas produções do Workshop de Informática na Escola. In: WORKSHOP DE INFORMÁTICA NA ESCOLA, 21., 2015, Maceió. Anais. Maceió: Sociedade Brasileira de Computação, 2015, p. 92-101.

MALTEMPI, M. V. Construção de Páginas Web: Depuração e Especificação de um Ambiente de Aprendizagem. Campinas: Faculdade de Engenharia Elétrica e de Computação, 2000. 197p. Tese de Doutorado.

MOTA, E. R. L. M. O Construcionismo de Papert como concepção epistemológica: fundamentos para qual educação?. Fortaleza: CED/UECE, 2014. 118p. Dissertação de Mestrado.

NUNES, S. C.; SANTOS, D. P. O Construcionismo de Papert na criação de um objeto de aprendizagem e sua avaliação segundo a taxionomia de Bloom. In: Encontro Nacional de Pesquisa em Educação em Ciências, 9., 2013, Águas de Lindóia. Atas. Águas de Lindóia: Associação Brasileira de Pesquisa em Educação em Ciências.

NUNES, S. C. As Dimensões de Papert e a Geometria Espacial: um estudo em laboratório de informática. In: ENCONTRO BRASILEIRO DE ESTUDANTES DE PÓS-GRADUAÇÃO EM EDUCAÇÃO MATEMÁTICA, 10, , 2016, Curitiba. Anais. Curitiba: Programa de Pós-Graduação em Ciências e em Matemática.

OSTI, A. Concepções sobre desenvolvimento e aprendizagem segundo a psicogênese piagetiana. Revista de Educação, São Paulo. v. 12, n. 13, p. 109-118, 2009.

PAPERT, S. Constructionism: A new opportunity for elementary science education. Cambridge: Massachusetts Institute of Technology, Media Laboratory, Epistemology and Learning Group, 1986.

SANTOS, J. T. G. A robótica educacional como metodologia de integração do currículo do ensino médio. Patos: CCEA/UEPB, 2014. 44p. Monografia.

THIOLlENT, M. Metodologia da Pesquisa-Ação. São Paulo: Cortez, 2004.

TSURUDA, L. K.; MENDES, T. A.; VITOR, L. R.; SILVEIRA, M. B. A importância da energia solar para o desenvolvimento sustentável e social. In: INTERNATIONAL WORKSHOP ADVANCES IN CLEANER PRODUCTION, 6., 2017, São Paulo. Anais. São Paulo: Programa de Pós-Graduação em Engenharia de Produção.

VALENTE, J. A. Informática na educação: instrucionismo $\mathbf{x}$ construcionismo. Educação Pública, $1997 . \quad$ Disponível em: <http://www.educacaopublica.rj.gov.br/biblioteca/tecnologia/0003.html>. Acesso: Março de 2017.

ZILLI, S. R. A Robótica Educacional no ensino fundamental: perspectivas e prática. Florianópolis: Universidade Federal de Santa Catarina, 2004. 89p. Dissertação de Mestrado. 